\title{
Toxic TikTok Trends
}

\author{
Ava Korbani ${ }^{1}$ and Jessica LaBrie ${ }^{1 \#}$ \\ ${ }^{1}$ Central Catholic High School, Lawrence, MA, USA \\ \#Advisor
}

\section{Abstract}

With the rise of social media, body image dissatisfaction has increased among young females and has led to a variety of negative consequences such as eating disorders. The TikTok app is the newest form of social media where users are encouraged to post creative videos and provide commentary. TikTok's ForYouPage, an algorithm that creates a symbiotic relationship between user interest in trends and a means to satisfy these interests, has promoted the longstanding theme of body image dissatisfaction on social media apps. The more users continue to express interest in toxic body trends, the more the app pushes this same content. My research will dive into the specific trends that appear on a TikTok ForYouPage, with the goal to determine the most toxic criteria prevalent on the TikTok body image atmosphere. For the purpose of my study, I created two separate equations: total measured toxicity (TMT) derived from a content analysis, and a total surveyed toxicity (TST) derived from a focus group survey. I concluded from the two equations that the act of giving or receiving qualitative or quantitative appearance rates is the most toxic criteria prevalent on TikTok's body image atmosphere. The results of this study give insight to how the app should take action to eliminate toxic body trends and prioritize restricting the most toxic criteria, as determined by my study.

\section{Literature Review}

The social media app, TikTok, has recently taken the world by storm. After reaching its first billion downloads in February 2019, the app received half a billion more in only eight months, and over two billion downloads as of April 2020 (Mohsin). This enormous user base, however, resulted in increased scrutiny from various groups, as well as a variety of negative consequences. One negative consequence specifically relates to advertisers and other social media influencers using exaggerated diet and fitness claims to promote unrealistic body goals. With $41 \%$ of users between the impressionable ages of 16 and 24, the app has developed numerous body-related content concerns (Aslam). These concerns would lead the app to release a new set of guidelines to address "problematic and exaggerated claims in diet and weight loss products" on the app (Rofagha).

TikTok provides a global platform for creative users to post a range of content from popular and original dance routines, to various short video productions. The content is under one minute and provides almost full artistic freedom. The short videos are only a part of the platform's overall content and entertainment, as users are encouraged to provide brief commentary on each video production. These comments are typically a sentence or two, and range from funny or positive, to negative and disturbing. Eager to grow in popularity on the app, TikTok influencers began posting videos glamorizing unrealistic body image and diet related goals. Flinders University senior lecturer and psychologist Ivanka Prichard notes that TikTok trends "idealize thinness and being skinny, and present people who appear to have no qualifications providing nutrition and fitness advice" (Dempster). One particular TikTok video analyzed by NBC News featured 17-year-old Kayla Long, who posted a video on TikTok commenting that she had only consumed a jug of water for the duration of the day, with the hashtag, what I eat in a day. This video received over 2 million views. Kayla, like most body image influencers on the app, are unqualified to give health related advice and are potentially unaware of the negative influence they inflict upon themselves, and their follower base. 
Despite the lack of credibility among most body related influencers, the TikTok user base continues to trust and blindly follow these trends. Maria-Irini Avgoulas, an Associate Lecturer in the School of Psychology and Public Health, La Trobe University, summarizes user interest in body related trends by noting how young people on social media, especially women, "will often blindly follow a trend if it can provide them with the qualities they desire, such as attractiveness and popularity, while disregarding the consequences to their health" (Verma). Kayla Long, who, as previously discussed, posted a video on TikTok commenting that she had only consumed a jug of water for the duration of the day, later revealed she was diagnosed with an eating disorder. Long revealed that "TikTok, along with other influences on social media like Instagram and Snapchat, had pushed her to restrict her food intake over the past year" (Kaufman). While Long was was at the height of her eating disorder, she continued to consume "several posts promoting unhealthy eating habits and disordered behaviors" due to content tracking and other algorithms used by the app meant to enhance the user experience (Kaufman). TikTok's ForYouPage is a tailored content page focused on specific user interests. As users, particularly young females, show inevitable interest in body trends, the app will search to continuously provide this same content. The app creates a symbiotic relationship between user interest in trends, and tailored content meant to satisfy these interests.

Due to social media apps' propensity to push body trends, eating disorders, like that of Kayla Long, and other unhealthy habits are not entirely unexpected. Rachel Cohen, a professor affiliated with the School of Psychology, Faculty of Science at University of Sydney, Camperdown, conducted a study to determine the relationship between body image dissatisfaction (BID), maintenance of eating disorders (ED) and appearance comparison (AC) on the app, Facebook. Results from a survey method provided that the use of social media sites, such as Facebook, put users at a greater risk of developing an eating disorder due to the "frequent exposure to thin- ideal content on Facebook [which] reinforces one's own body- related concerns, eliciting cognitive biases that lead one to selectively attend to thin-ideal content on Facebook" (Cohen). Social media apps, like TikTok's ForYouPage, continue to push almost endless options of body related content, which, according to Cohen, can result in eating disorders. As collectively demonstrated by Cohen and the Kayla Long example, frequent exposure to thin ideal content can increase the likelihood of bodyrelated issues such as eating disorders.

While Prichard, Cohen, and Avogulas worked to describe the reasoning behind the popularity of social media trends and their general, negative consequences, they never researched which specific social media trend most greatly contributed to their findings. Additionally, the aforementioned social media research was focused on older, more established apps, like Facebook and Instagram. Given the relatively recent and meteoric rise of TikTok, no real, specific research has been performed on the TikTok app. Thus, my research will be one of the first to contribute to the scholarly conversation regarding TikTok as an individual app. In response to early-stage issues relating to body toxicity, TikTok chose an extremely narrow view of the body toxicity issue. TikTok leadership chose to ban all diet and weight-loss paid advertisements from the app, concluding that paid sponsors were responsible for negative trends and body related issues. TikTok never looked to their own user base and influencers to determine which trends most greatly contributed to the need for their new set of guidelines. Current knowledge surrounding TikTok's body related content only focuses on the general, negative impact resulting from these trends, holistically. There is no current analysis related to individual, specific trends and their respective negative consequences on TikTok's female user base. This gap relating to unknown and specific user created trends led me to the question: Which specific TikTok trend and corresponding criteria most greatly contributes to the toxicity surrounding TikTok's body image atmosphere? By studying specific, toxic TikTok trends, I am studying what Cohen, Prichard, Avgoulas, and TikTok failed to acknowledge: specific and individual trend toxicity surrounding body culture atmospheres.

Given the research by Cohen surrounding eating disorders as a consequence of consistent toxic trend consumption, I hypothesized that the most toxic trends would be relating to food consumption. By using a mixed method approach, I would be able to prove, through a content analysis and survey, if this hypothesis was in fact true. I chose a mixed method approach because it was the most effective way to collect two variations of targeted data: toxic trend popularity $\&$ human opinion regarding toxic trends. By conducting a content analysis on the app, itself, I would best be able to 
gather specific data regarding trend popularity. Additionally, a focus group survey would supplement the content analysis with a human element and personal perspective.

\section{Methods}

\section{Overview}

This study is a mix of two methods: content analysis \& focus group survey. There are 3 main steps in my content analysis method: I first select toxic trends based on a predetermined toxic criteria chart, to collect and analyze from TikTok. I then analyze and record the number of likes, shares, views, followers, and comments in relation to each other per video trend. Lastly, I measure the total measured toxicity (TMT) from my derived equation. There are 4 main steps in my second method of a focus group survey: I fist send a survey asking for voluntary female focus group participants in the Merrimack Valley. Next, I choose an example of each TikTok trend to show focus group participants. Then, I conduct a quantitative survey in relation to the trends through focus group participants. Lastly, I measure the total surveyed toxicity (TST) from my second derived equation.

\section{Different Trends}

In this study, I selected and compared six TikTok trends and corresponding criteria which reflected the toxic atmosphere of TikTok's body image and awareness culture. In order to determine the toxic criteria, I would analyze in my study, I created a new TikTok account called @akorbaniapresearch. I created this account because it would provide an unbiased ForYouPage, in which I "liked" videos I believed reflected toxic body related criteria. Because of TikTok's ForYouPage algorithm, the app then pushed more body related content that I was able to watch and group into six overarching criteria and corresponding trends. I picked six criteria because it allowed me to gauge a diverse population of trends that present differing levels of toxicity to female users. Since the TikTok app is constantly evolving and pushing new content, my study focuses on the toxic criteria most prevalent on TikTok's body culture atmosphere. While trends are constantly changing, the toxic criteria remain constant and can be matched to new and evolving trends. A limitation of my research is that I wasn't able to analyze all of TikTok's toxic criteria due to time constraints. However, the six chosen toxic criteria represent a solid foundation that remains relevant to many new evolving TikTok trends. By deriving Figure 1, I was able to define the toxic criteria I witnessed through my unbiased TikTok account used for the purpose of this study. Figure $\mathbf{2}$ defines each matched trend per criterion and how users create TikTok content relating to that trend.

Figure 1. Toxic Criteria

\begin{tabular}{|l|l|c|}
\hline & Toxic Criterion & Corresponding Trend \\
\hline 1 & Encourages unhealthy, restricted, or & Trend 1: What I Eat in a Day \\
& heightened food intake. Promotes & \\
& unsustainable diets and encourages & \\
& disordered eating & \\
\hline
\end{tabular}




\begin{tabular}{|c|c|c|}
\hline 2 & $\begin{array}{l}\text { Advocates unrealistic exercise } \\
\text { goals aimed toward body slimming }\end{array}$ & Trend 2: Workout Routine \\
\hline 3 & $\begin{array}{l}\text { Seeking validation through a sys- } \\
\text { tem of qualitative or quantitative } \\
\text { rates from the TikTok user base }\end{array}$ & Trend 3: Rate My Appearance \\
\hline 4 & $\begin{array}{l}\text { Glamorizes societal norms regard- } \\
\text { ing petite body figures }\end{array}$ & Trend 4: So You Think I'm Skinny \\
\hline 5 & $\begin{array}{l}\text { Unwilling quantitative or qualita- } \\
\text { tive appearance rates from the Tik- } \\
\text { Tok user base }\end{array}$ & Trend 5: Rating TikTok Girls \\
\hline 6 & $\begin{array}{l}\text { Glamorizes thinness and curvy } \\
\text { body figures in a showy and for- } \\
\text { mally presented fashion }\end{array}$ & Trend 6: Hourglass Challenge \\
\hline
\end{tabular}

Figure 2. Criteria Explanation

Trend Number

Trend

Trend Explanation 


\begin{tabular}{|c|c|c|}
\hline 1 & What I Eat in a Day & $\begin{array}{l}\text { Users post their malnourished or } \\
\text { binged daily food and calorie intake } \\
\text { to catchy songs, encouraging others } \\
\text { to follow }\end{array}$ \\
\hline 2 & Workout Routine & $\begin{array}{l}\text { Videos highlighting unrealistic ex- } \\
\text { ercise routines and goals: Ex: "abs } \\
\text { in a day" }\end{array}$ \\
\hline 3 & Rate My Appearance & $\begin{array}{l}\text { Users post a video of themselves, } \\
\text { asking viewers to quantitatively or } \\
\text { qualitatively rate their appearance } \\
\text { in terms of plainness, ugliness, or } \\
\text { attractiveness. }\end{array}$ \\
\hline 4 & So You Think I'm Skinny & $\begin{array}{l}\text { Creators post common scenarios } \\
\text { glamorizing societal norms such as } \\
\text { petite clothing sizes in relation to } \\
\text { thin people. Ex: celebrating a sales } \\
\text { associate bringing a smaller size } \\
\text { clothing than the customer is typi- } \\
\text { cally }\end{array}$ \\
\hline 5 & Rating TikTok Girls & $\begin{array}{l}\text { Creators post videos with pictures } \\
\text { of unrelated women, and give them } \\
\text { a numerical score from } 1-10 \text { based } \\
\text { on attractiveness. Ex: middle aged }\end{array}$ \\
\hline
\end{tabular}




\begin{tabular}{|c|c|c|}
\hline & & $\begin{array}{c}\text { men rate 16-year-old females, } \\
\text { based on appearance }\end{array}$ \\
\hline 6 & Hourglass Challenge & $\begin{array}{c}\text { Female users stand in baggy } \\
\text { TeeShirts to the song "Bang Bang" } \\
\text { by Jessie J, Ariana Grande and } \\
\text { Nikki Minaj. At the point in the } \\
\text { song "she got a body like an hour- } \\
\text { glass," creators pull back their } \\
\text { baggy clothing to reveal their hour- } \\
\text { glass shape and curved figure }\end{array}$ \\
\hline
\end{tabular}

These trends (Figure 2) were chosen because they embodied criteria 1-6 respectively (Refer to Figure 1 for criteria explanation).

\section{Gathering Content}

After finalizing six trends and criteria to evaluate in my study, I selected 17 videos of each trend to include in my content analysis, totaling 102 videos. Due to time constraints and the immense amount of TikTok videos per trend, I was limited to the number of videos I could evaluate per trend. I chose 17 videos per trend to yield a total video count of over 100 TikTok posts, which produced a sufficient amount of data for my content analysis. The following conditions were applied for each video post selected: The video must not show any inappropriate content, which would allow my study to stay school appropriate and be approved for focus group participants. The post must be over one week old, allowing the video to reach the potential of its popularity and growth. The video must have interaction: likes, shares, \& views, to fulfill my content analysis' variables. Lastly, the post must reflect the specific criteria of the trend (Figure 1).

Given TikTok's assortment of creators and immense popularity, not every single video per trend reflected toxic criteria. Often, creators would adjust the toxic trend to make it lighthearted and advocating for positive ideas. However, given that the purpose of my study focuses around the toxicity of TikTok's body image atmosphere, I analyzed TikTok videos that reflected the toxic criteria previously established in Figure 1. Videos reflecting the toxic criteria per trend were the majority of my findings. Further research can be conducted regarding the minority of the positive adapted version per trend.

The mediums used to gather trend samples varied slightly. For trends 1,2,4,5,6, videos were pulled from their respective hashtag pages. Each hashtag on TikTok has its own search page with videos specifically captioned with the pertaining hashtag. The hashtag medium for collecting TikTok posts showed a representative and easy way to access direct, labeled posts in relation to the trend, and was the most effective medium of video collection for trends 1,2,4,5,6. On the contrary, trend 3 videos were collected from one of TikTok's sound pages. Sound pages on TikTok show every public post that uses the same soundtrack. The \#ratemyappearance page totaled only two videos posted under the 
trend, for the trend centered around a common soundtrack audio, not a hashtag. Trend 3 included in my study always used the soundtrack "Ultralight Beam," by Chance the Rapper. The sound page "Ultralight Beam" by creator @jenxxtra totaled $24.3 \mathrm{k}$ videos posted with the sound, and thus served as a more effective medium for trend 3 video collection.

\section{Data Equations and Synthesis}

After collecting 17 videos per trend that met my research requirements, I performed a content analysis on each trend. My content analysis yielded quantitative averages for four variables: The first variable was the number of characterized toxic comments $(C T C)$ in the top five most liked comments. The next variable was the number of likes per the number of views $(L P V)$. Another variable was the number of shares per the number of views $(S P V)$. The final variable was the number of views per the number of followers $(V P F)$. The average of these categories yielded the trend's total measure of toxicity (TMT) (Figure 3). I derived the following equation.

Figure 3: TMT Equation

$$
T M T=\frac{C T C+L P V+S P V+V P F}{4}
$$

This equation (Figure 3) was derived specifically to format and fulfill my research question based on specific toxic trend popularity. Each variable in the TMT equation contributes to determining the overall numerical value of toxicity for the purpose of my study. CTC was chosen because it is a direct representation of negative interactions on specific trends and their posts. I created the following CTC condition and example figure to communicate the conditions for which comments were included in the CTC. By establishing a common CTC criteria chart, (Figure 4) my overall CTC comparison between trends would be constant. I derived the following criteria chart (Figure 4).

Figure 4: CTC Criteria Chart

\begin{tabular}{|c|c|c|}
\hline CTC Criteria & Conditions & Example \\
\hline $1 \mathrm{C}$ & $\begin{array}{l}\text { Reflects an abrasive, insulting, or } \\
\text { critical tone towards the creator }\end{array}$ & "ur the ugliest person I've seen " \\
\hline $2 \mathrm{C}$ & $\begin{array}{l}\text { Reflects a self-doubting, insulting, } \\
\text { or demeaning tone towards oneself }\end{array}$ & $\begin{array}{l}\text { "Does this really work I cry every } \\
\text { night because all these girls are } \\
\text { skinny and I'm not" }\end{array}$ \\
\hline $3 \mathrm{C}$ & $\begin{array}{l}\text { Contributed to the overall trend and } \\
\text { reflects agreement with the toxic el- } \\
\text { ement }\end{array}$ & $\begin{array}{l}\text { "Below Average"(response to trend } \\
\text { 3) }\end{array}$ \\
\hline
\end{tabular}


Given the popularity of most of the videos included in the content analysis, there were often thousands of comments on specific videos. My research is limited in the sense that I was not able to analyze every toxic comment per video, however, sampling the five top liked comments through Figure 4, per video, allowed for an accurate representation of what the rest of the comment section would reflect.

The $L P V$ variable was chosen because it reflects the average popularity of the specific trend, in relation to the number of likes. The number of likes per total views for the particular toxic video demonstrates how many people enjoyed or supported the toxic content provided by the creator. The SPV variable was chosen because it shows a further representation and indicator of each toxic TikTok trend's user popularity. The share feature allows users to send the toxic post to others on TikTok, text, or even email. The higher the $L P V \& S P V$ percent, the more people who watched the toxic post decided to like or share it, thus increasing its audience reach, popularity, and consequently increasing its TMT.

The VPF variable was chosen because it reflects the popularity the post gained on TikTok's ForYouPage. A greater $V P F$ value indicates a higher volume in which TikTok is distributing the toxic trend. The higher the $V P F$, the more responsible TikTok is for distributing and promoting the toxic trend.

Finally, the $T M T$ is an average of $C T C, L P V, S P V$, and $V P F$ for each trend. The higher the $T M T$, the more toxic the trend presents to be. I decided to make the $T M T$ an average of the four variables to provide an effective method of comparison between the trends as a whole.

See Appendix A for collected data charts.

Focus Group Survey Method

The second part of my method includes focus groups conducted at my school. This part of my method was included because it would allow for specific human opinions in relation to the TMT derived in my content analysis, which was based on toxic trend popularity. Conducting a focus group would allow me to gather teenage girl's specific perspectives on demonstrated TikTok trends and criteria. I gathered focus group participants by sending an email survey to my school, asking for female volunteers to participate. Given that the purpose of my study was to examine the teenage female perspective of TikTok's body image atmosphere, those who identified as female were able to participate. I chose to focus on the female perspective because the six trends in my study have a majority of female creator interaction and marketing. When prompted with the option of volunteering for a focus group, survey participants were made aware that participation was completely anonymous and voluntary. Students were not presented with focus group questions or videos prior to the session, and no prize was offered to the students for participation. In total, there were 15 participants. This number may serve as a limitation, for I was limited as to the number of participants willing to take part in my focus group. However, the 15 participants served as an adequate media for data. My AP Research teacher was present during my focus group meetings and did not participate in any discussion or survey. During the focus group, I showed six TikTok posts, each representing a different trend, followed by three specific questions per trend.

\section{Focus Group Videos Presented}

Six predetermined TikTok video posts were chosen to be presented to focus group participants. Each video reflected a different trend, and was chosen from the 102 TikTok videos in my content analysis. Figure 5 communicates the specific videos and creator posts shown to the focus group. 
Figure 5. Focus Group Presented Posts

\begin{tabular}{|c|c|c|}
\hline Trend Number & Creator & Video Explanation \\
\hline 1 & @cassidyflood23 & $\begin{array}{l}\text { Presented videos of food consumed } \\
\text { throughout the day including the } \\
\text { calorie count: } 1089 \text { ( } 50 \% \text { less than } \\
\text { the average recommended } 2,200 \\
\text { daily calories for adolescent fe- } \\
\text { males). }\end{array}$ \\
\hline 2 & @marriedtomathis & $\begin{array}{l}\text { Highlighted workout regimens spe- } \\
\text { cifically focused on a "smaller } \\
\text { waist and toned abs." }\end{array}$ \\
\hline 3 & @divineraposo & $\begin{array}{c}\text { Creator asked viewers to rate her } \\
\text { appearance based on the adjectives } \\
\text { "plain, average, or pretty." }\end{array}$ \\
\hline 4 & @mynameisalex.french & $\begin{array}{l}\text { Creator celebrating that her mom } \\
\text { bought her a smaller clothing size. }\end{array}$ \\
\hline 5 & @natalieshaw_ & $\begin{array}{l}\text { An older woman giving adolescents } \\
\text { a numerical value based on appear- } \\
\text { ance attractiveness. }\end{array}$ \\
\hline 6 & @apriltiberc & $\begin{array}{l}\text { Showed the user flaunting her hour- } \\
\text { glass and curved body figure. }\end{array}$ \\
\hline
\end{tabular}

Each of the six videos was chosen specifically because they fulfill their predetermined toxic criteria as expressed in Figure 1 and would serve as adequate video samples for my focus group survey questions. 


\section{Focus Group Questions}

After each video was formally presented on screen and played twice, focus group participants were asked to complete three questions on a corresponding survey that went along with the trend. Participants filled out the questions right after watching the specific trend video rather than at the end of all the videos because it would reflect a more accurate and uninfluenced reaction in their answers. The survey students filled out during the focus group was 22 questions long: 3 preliminary questions, 18 questions directly related to the videos (three questions per trend), and an optional section for comments at the end. The three questions per trend video were relatively the same and based on the same goal in relation to each other. Figure 6 shows the explanations and guidelines of each question asked per trend.

Figure 6. Survey Statement Baseline

\begin{tabular}{|c|c|c|}
\hline Survey Question & Explanation & Example \\
\hline A1 & focused specifically around the & the presented video made you feel \\
& toxic criteria per each trend & negatively about your [specific cri- \\
\hline A2 & focused on general terms of body & the presented video negatively im- \\
& image & $\begin{array}{c}\text { pacted yourself perception in terms } \\
\text { of body image }\end{array}$ \\
\hline A3 & focused on an external opinion & $\begin{array}{c}\text { the presented video demonstrates a } \\
\text { toxic trend that may have negative }\end{array}$ \\
\hline
\end{tabular}

Participants were asked to numerically rate on a scale of 1-10, 1 being not at all and 10 being very much, for each prompted statement in the survey based on A1, A2, \& A3. A1 (Figure 6) for each trend was the only differing question between the trends and was included to observe and gather data on how specific elements and criteria of TikTok trends relate to and reflect toxic reactions and on the app. The purpose of A2 (Figure 6) was to gather data on how the specific trends affected user body image on a larger scale. Differing from A1 which provided specific reactions to each trend, A2 provides for a more generalized representation of the criterion's toxicity in terms of body image on the app and would provide for a unified comparison between the trends as a whole. A3's (Figure 6) purpose was to gauge how teenage girls perceived the app's body image atmosphere externally and holistically. By not specifically targeting the participants internal and self-opinions on the trend, I was hoping to collect more honest feedback. While participants may have been hesitant to note their true emotions in relation to themselves for A1 \& A2, A3 allowed participants to express how they felt the trend contributed to TikTok's toxic body culture. I then found the averages of A1, $\mathrm{A} 2, \& \mathrm{~A} 3$ per trend from the 1-10 scale, and added the three averages of A1, A2, \& A3, to derive my own equation yielding the total surveyed toxicity (TST) per trend (Figure 7). 
Figure 7. TST Equation

$$
A 1+A 2+A 3=T S T
$$

I derived this equation to supplement and provide for comparison of my content analysis' TMT. The equation for TST demonstrates the average surveyed toxicity per each trend. The higher the TST, the more toxic teenage girls perceive the trend to be. This TST variable, which represents the perspective of teenage girls in the Merrimack Valley, provides for effective synthesis and comparison in relation to my content analysis' TMT. By collecting these two averages, I would be able to support and compare my findings between my two methods to determine the trend and corresponding criteria that most greatly targets TikTok's body image atmosphere.

See Appendix $B$ for data charts in relation to $A 1, A 2, \& A 3$ per video \& survey questions.

\section{Results}

\section{Content Analysis Results}

The following results demonstrate the variable and total results of my content analysis' equation (Figure 3). The calculations shown in Figure 8 include the CTC, LPV, SPV, VPF, and TMT.

See Appendix $C$ for variable chart comparison

Figure 8. Content Analysis Data

\begin{tabular}{|c|c|c|c|c|c|}
\hline & $C T C$ & $L P V$ & $S P V$ & $V P F$ & $T M T$ \\
\hline $\begin{array}{c}\text { What I Eat in a } \\
\text { Day }\end{array}$ & 2.8 & $17.2 \%$ & $0.2 \%$ & 61.56 & 16.1 \\
\hline $\begin{array}{l}\text { Workout Rou- } \\
\text { tine }\end{array}$ & 3.1 & $16.8 \%$ & $1.8 \%$ & 95.64 & 24.7 \\
\hline $\begin{array}{c}\text { Rate My Ap- } \\
\text { pearance }\end{array}$ & 4.3 & $12.5 \%$ & $0.1 \%$ & 325.13 & 82.4 \\
\hline $\begin{array}{l}\text { So You Think } \\
\text { I'm Skinny }\end{array}$ & 1.8 & $13.7 \%$ & $0.1 \%$ & 101.49 & 25.9 \\
\hline $\begin{array}{c}\text { Rating TikTok } \\
\text { Girls }\end{array}$ & 3.6 & $12.7 \%$ & $0.2 \%$ & 76.81 & 20.1 \\
\hline
\end{tabular}




\begin{tabular}{|c|c|c|c|c|c|}
\hline $\begin{array}{c}\text { Hourglass Chal- } \\
\text { lenge }\end{array}$ & 3.2 & $10.7 \%$ & $0.1 \%$ & 52.69 & 14.0 \\
\hline
\end{tabular}

Figure 8 reports the variables derived in my content analysis equation (Figure 3). Trend 3 had the highest average $C T C$, averaging 4.3 toxic deemed comments in the top five comments. Trend 5 was the next highest with a 3.6 CTC. The third highest was trend 6 with a CTC of 3.2 (Appendix C Figure 12). Trend 1 had the highest $L P V$ percent, averaging $17.2 \%$ of the number of viewers who liked the video. The next highest $L P V$ value was trend 2 with a $16.8 \%$ $L P V$. The third highest $L P V$ percentage was trend 4, with a $L P V$ of $13.7 \%$ (Appendix $C$ Figure 13). For the $S P V$ variable, trend 2 greatly surpassed the other trend's $S P V$ percentages, with a $S P V$ of $1.8 \%$. The next highest $S P V$ percent was trends $1 \& 5$, tied at .2\% SPV (Appendix $C$ Figure 14). The highest $V P F$ value belonged to trend 3, with a $V P F$ value of 325.13. The next highest $V P F$ value belonged to trend 4, with a value of 101.49. Trend 2 followed next with a $V P F$ of 95.64 (See Appendix C Figure 15). Figure 9 shows the TMT data comparison chart.

\section{Total Measure of Toxicity Content Analysis}

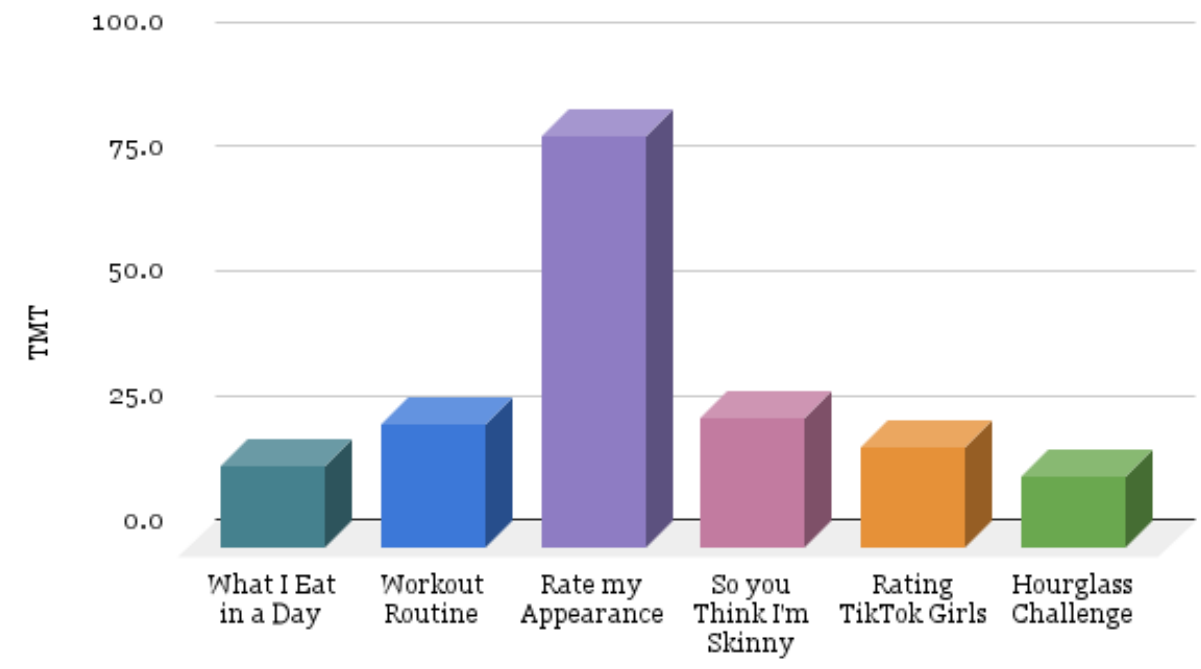

Figure 9. Total Measure of Toxicity (TMT) Chart

Figure 9 shows the results of the equation for TMT derived in my content analysis (Figure 3). The highest TMT value was trend 3 with a $T M T$ of 82.4 , largely due to its high $V P F$, indicating that TikTok was greatly encouraging and spreading the toxic trend. The second highest TMT of 25.9 came from trend 4 . The third highest TMT of 24.7 belonged to trend 2. Evaluating the TMT was critical for my research to collaborate with my focus group survey results to determine which TikTok trend and criteria most greatly contributes to TikTok's toxic body atmosphere (Refer to Figure 8 for specific $T M T$ values).

Focus Group Survey Results

Figure 10 shows the data collected from my focus group survey. The calculations shown include the variables $A 1$, $A 2, A 3$, and total surveyed toxicity (TST) (See Figure 7 for equation).

Figure 10. Focus Group Data 


\begin{tabular}{|c|c|c|c|c|}
\hline & $\begin{array}{c}\text { Question 1: Specific } \\
\text { (A1) }\end{array}$ & $\begin{array}{c}\text { Question 2: Body } \\
\text { Image (A2) }\end{array}$ & $\begin{array}{l}\text { Question 3: Others } \\
\text { (A3) }\end{array}$ & TST \\
\hline What I Eat in a Day & 4.93 & 3.40 & 6.00 & 14.33 \\
\hline Workout Routine & 6.47 & 6.33 & 6.60 & 19.40 \\
\hline $\begin{array}{c}\text { Rate My Appear- } \\
\text { ance }\end{array}$ & 5.13 & 5.47 & 8.87 & 19.47 \\
\hline $\begin{array}{c}\text { So You Think I'm } \\
\text { Skinny }\end{array}$ & 4.53 & 4.80 & 6.20 & 15.53 \\
\hline Rating TikTok Girls & 8.80 & 6.13 & 8.87 & 23.80 \\
\hline $\begin{array}{c}\text { Hourglass Chal- } \\
\text { lenge }\end{array}$ & 6.13 & 5.93 & 8.67 & 20.73 \\
\hline
\end{tabular}

Trend 5 had the highest $A l$ average of 8.80. Trend 2 had the next highest $A l$ average of 6.47 . The third highest $A l$ average of 6.13 belonged to trend 6 . For $A 2$, trend 2 had the highest average of 6.33 . Trends $5 \& 6$ followed behind with $A 2$ averages of 6.13 and 5.93, respectively. Trends $3 \& 5$ tied for the highest $A 3$ value of 8.87 . The next highest $A 3$ value of 8.67 belonged to trend 6 . Figure 11 shows my TST comparison chart.

\section{Total Surveyed Toxicity (TST)}

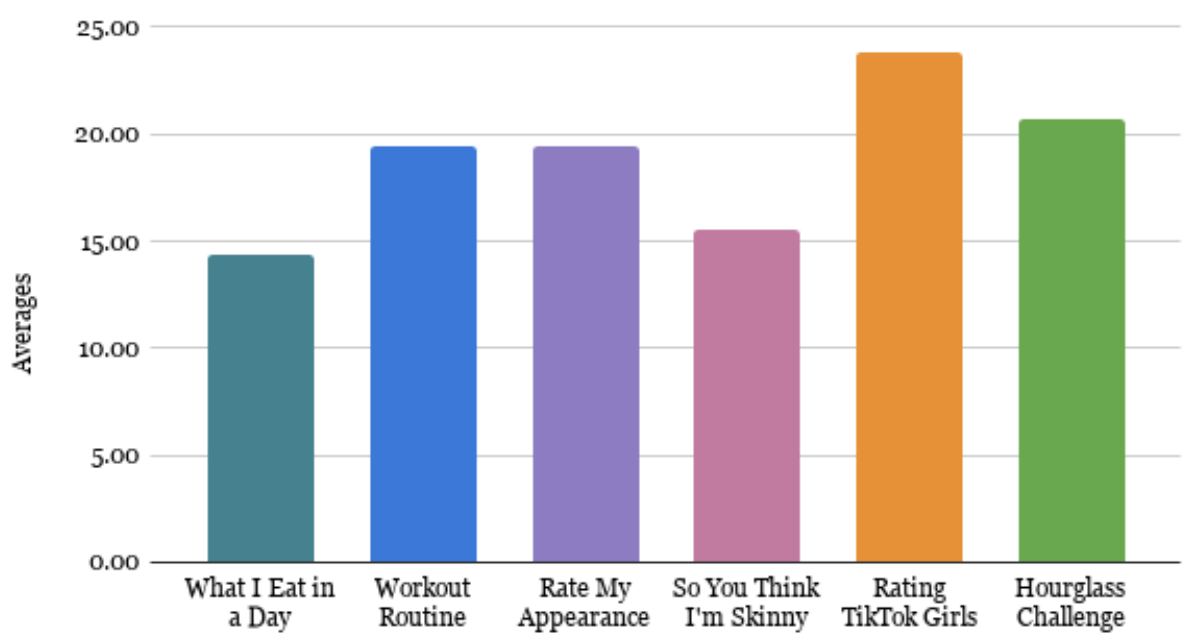




\section{Figure 11. Total Surveyed Toxicity (TST) Comparison}

Figure 11 shows the comparison and final results of the equation: $A 1+A 2+A 3=T S T$, derived in my method section (Figure 7). Trend 5 had the highest TST of 23.80. The next highest TST of 20.73 belonged to trend 6. The third highest TST of 19.47 belongs to trend 3 .

Besides quantitative data, my focus group survey presented qualitative data in the form of an optional comment section at the end of the survey. This qualitative data would further add a human element and specific trend feedback that would supplement quantitative survey responses. One participant noted "these trends can negatively impact an impressionable group of girls, who may already be insecure in their bodies." Similarly, another participant noted, "TikTok is a platform with an enormous demographic; ED (eating disorder) survivors, people currently battling EDs, and generally very self-conscious people are all among that demographic.” As previously established, Kayla Long also suffered from an eating disorder and fell into this category of being affected by toxic trends in relation to food intake and body insecurity. This comment enforces the fact that young, impressionable teenagers are consuming these toxic trends which have detrimental consequences to their body awareness. Thus, my research is essential to determine which trend most greatly influenced the toxicity surrounding TikTok body culture that encourages eating disorders like that of Long. This feedback enforces the fact that the primary audience of these toxic trends are young adolescent girls. My research is needed to protect the female TikTok user base against toxic criteria that could negatively impact their self perception in terms of body image. Given that another participant noted "TikTok targets those who are already vulnerable to criticism and have low self esteem and makes it worse," there needs to be a change in the way TikTok approves and pushes content related to female body culture. Thus, enforcing the need to address the gap in knowledge regarding user created trends that contribute to body atmosphere toxicity.

\section{Discussion \& Conclusion}

The results of my content analysis and focus group survey highlighted a specific trend type that was clearly the most toxic. Trend 3 had the highest scored TMT and third highest scored TST. Thus, it can be inferred through this study that out of the six evaluated trends and criteria, criteria \& trend 3 (Rate My Appearance), most greatly contributes to the toxicity surrounding TikTok's body atmosphere. Additionally, the highest scored TST was trend 5, Rating TikTok Girls. It is valuable to note that the two highest TMT and TST values came from criteria 3 and 5, respectively. These two criteria represent a very similar criteria type, which is receiving or giving "qualitative or quantitative rates" based on appearance (Figure 1) from the TikTok user base. Thus, it is reasonable to assume that the act of seeking or receiving a form of qualitative or quantitative appearance rate is the most toxic criteria prevalent in TikTok's body image atmosphere.

Given previous learnings from Avogulas related to following desired trends, combined with my research on eating disorders like that of Long, I hypothesized trend 1 would score most toxic. Trend 1 represented a repeatable trend relating to unrealistic food consumption that users, primarily young girls, could easily follow and replicate. However, trend 1 had the lowest TST and seconds lowest TMT. This could be due to the fact that my previous learning relating to trend 1 was more focused on negative outcomes, such as eating disorders, and not a pure toxicity score. This presents a concerning implication, for the least toxic trend in my study has a known consequence of eating disorders, yet the implications of trends $3 \& 5$ remain unknown. Further research is needed to investigate the specific effects and negative outcomes that nonfood related posts have on TikTok's user base, specifically the psychological implications of posts relating to criteria $3 \& 5$.

Though trends 3 and 5 presented criteria that proved to be the most toxic, they weren't the only trends analyzed in this study. Trends 1,2,4,6 each proved to demonstrate traits of toxic trends, yet on a lower scale than that of trends 3 and 5. All of my research led to the conclusion that the TikTok trend that most greatly contributes to the toxicity surrounding TikTok's body culture, is any trend that can be identified as criteria 3 or 5, or involves "qualitative or quantitative" appearance rates. Thus, I filled the knowledge gap in research regarding the TikTok criteria that most greatly 
influenced the toxicity surrounding TikTok's body image atmosphere. Though there have been numerous studies surrounding body culture on more established social media apps, like Rachel Cohen's Facebook study, my research will be one of the first to add to the scholarly conversation regarding TikTok as an individual app. Thus, I filled a general knowledge gap surrounding the lack of TikTok specific research, for my research serves as a catalyst and beginning to future TikTok specific research. Additionally, my research filled in the gap regarding the reasoning and supplemental cause to TikTok's released statement on body positivity. My research established that the real toxicity surrounding TikTok's body culture was evident in user trends most specifically relating to the act of giving or receiving qualitative or quantitative appearance rates. My research is limited in the sense that I was not able to analyze every criterion and corresponding trend on TikTok's content platform. Given that the TikTok app is constantly evolving and pushing new trends, the trends chosen for the purpose of my study are variable. However, my established criteria chart (Figure 1), can be adapted and fitted to TikTok's new and evolving toxic body content. Further research can be conducted on possibly new evolving criteria not listed on my criteria chart, and synthesized with my own observed toxic criteria (Figure 1). For the purpose of further research, I recommend combining criteria 3 and 5 into one toxic criterion. Though criteria 3 and 5 being separated allowed me to clearly see the magnitude of toxicity regarding "qualitative or quantitative appearance rates," further research can combine the two criteria, for their individual toxicity has already been established through my study.

Rather than simply releasing a statement regarding their stance on body positivity and banning weight loss advertising, TikTok should have looked to their own user base and made strides to eliminate user created trends that appear harmful to viewers. Implications of my research regarding the toxicity of the app's body trends should lead the app in the right direction towards making these strides to eliminate body toxicity, starting with addressing any post or commentary related to appearance rates. The app could start deciding to ban content relating to criteria $3 \& 5$, however, this presents the possibility of infringement of the app's esteemed freedom of expression and creativity. TikTok's response depends on how badly they want to protect their vast, young, and impressionable audience against toxic trends, and consequently, promote a positive body image platform.

\section{Acknowledgements}

I'd like to thank my teacher, Mrs. LaBrie, for supporting and guiding me throughout the process.

\section{References}

Aslam, Salman. “Omnicore Agency - Digital Marketing Agency.” OmnicoreAgency.com, 11 Feb. 2020. www.omnicoreagency.com/tiktok-statistics/.

Bowenbank, Starr. “Charli D'Amelio and Addison Rae Clapped Back at Body Shamers on Twitter for Criticizing Their Weight." Cosmopolitan, 28 Apr. 2020. https:/www.cosmopolitan.com/lifestyle/a32303772/charlidamelio-addison-rae-clapback-twitter-weight-body-shamers/

Chen, Tanya. "Young Women Are Jumping On A Viral TikTok Chain Where They Dance With Their Stomachs Out." BuzzFeed News, 9 Sept. 2020. https://www.buzzfeednews.com/article/tanyachen/influencers-viralbody-positive-tiktok-chain

Cohen, Rachel, and Alex Blaszczynski. "Comparative effects of Facebook and conventional media on body image dissatisfaction." Journal of Eating Disorders, vol. 3, no. 1, 2015. Gale OneFile: Gender Studies, link.gale.com/apps/doc/A541671770/PPGB?u=mlin_n_cchs\&sid=PPGB\&xid=c105b3ab. 
Dempster, Alice. ''Extremely Unsafe': TikTok Videos Fuelling Eating Disorders amid Coronavirus Lockdowns, Experts Warn.” ABC News, 12 May 2020. https://www.abc.net.au/news/2020-05-13/experts-concernedtiktok-content-fuelling-eating-disorders/12215986

De Sousa Silva, Ana Flávia, et al. "Image-Discursive Construction of Body Beauty in Social Media: Effects on Followers' Body and Eating Perceptions." Demetra: Food, Nutrition \& Health / Alimentação, Nutrição \& Saúde, vol. 13, no. 2, June 2018, pp. 395-411. EBSCOhost. https://doi.org/10.12957/demetra.2018.33305

Dubicka, Bernadka, et al. "Editorial: Screen Time, Social Media and Developing Brains: A Cause for Good or Corrupting Young Minds?" Child \& Adolescent Mental Health, vol. 24, no. 3, Sept. 2019, pp. 203-204. EBSCOhost. https://doi.org/10.1111/camh.12346

Fardouly, Jasmine, et al. “Instagram Use and Young Women's Body Image Concerns and Self-Objectification: Testing Mediational Pathways." Sage, New Media \&amp; Society 2018, Vol.20(4) 1380-1395. http://www2.psy.unsw.edu.au/Users/lvartanian/Publications/Fardouly,\%20Willburger,\%20\%26\%20Vartani an\%20(2018).pdf

Feltman, Chandra E., and Dawn M. Szymanski. "Instagram Use and Self-Objectification: The Roles of Internalization, Comparison, Appearance Commentary, and Feminism.” Sex Roles, vol. 78, no. 5-6, Mar. 2018, pp. 311-324. EBSCOhost. https://doi.org/10.1007/s11199-017-0796-1

Hanley, Sarah M., et al. "Taking a break: The effect of taking a vacation from Facebook and Instagram on subjective well-being." Plos One, vol. 14, no. 6, 2019, p. e0217743. Gale Health and Wellness. link.gale.com/apps/doc/A587945346/HWRC?u=mlin_n_cchs\&sid=HWRC\&xid=a333d3c0.

Kaufman, Sarah. "'It's Not Worth It': Young Women on How TikTok Has Warped Their Body Image." NBCNews.com, NBCUniversal News Group, 19 July 2020. https://www.nbcnews.com/tech/tech-news/it-snot-worth-it-young-women-how-tiktok-has-n1234193

Köver, Chris. "Discrimination - TikTok Curbed Reach for People with Disabilities.” 2 Dec. 2019. https://netzpolitik.org/2019/discrimination-tiktok-curbed-reach-for-people-with-disabilities/

Lim, Yujin, and Soontae An. "Effects of Attributions and Social Media Exposure on Obesity Stigma Among Korean Adolescents." Social Behavior and Personality: An International Journal, vol. 46, no. 12, 2018, p. 2049+. Gale Health and Wellness. link.gale.com/apps/doc/A567425986/HWRC?u=mlin_n_cchs\&sid=HWRC\&xid=c723a82e

McLean, Sian A., et al. "How do 'Selfies' Impact Adolescents' Well-Being and Body Confidence? A Narrative Review." Psychology Research and Behavior Management, 2020, p. 513c+. Gale OneFile: Psychology. link.gale.com/apps/doc/A622150966/PPPC?u=mlin_n_cchs\&sid=PPPC\&xid=e8e480ea.

Mohsin, Maryam. "10 TikTok Statistics You Need to Know in 2021 [March Data].” Oberlo, 30 Apr. 2021, https://www.oberlo.com/ 
Ormsby, Hollie, et al. "A Brief Report on the Associations Amongst Social Media Use, Gender, and Body Esteem in a UK Student sample." Current Psychology, vol. 38, no. 2, 2019, p. 303+. Gale OneFile: Psychology. link.gale.com/apps/doc/A582798188/PPPC?u=mlin_n_cchs\&sid=PPPC\&xid=e3b76e05

Peiró-Velert, Carmen, et al. "Screen Media Usage, Sleep Time and Academic Performance in Adolescents: Clustering a Self-Organizing Maps Analysis.” Plos One, vol. 9, no. 6, June 2014, pp. 1-9. EBSCOhost. https://doi.org/10.1371/journal.pone.0099478

Porterfield, Carlie. "TikTok Takes On 'Body Shaming' With A Ban On Ads For Fasting Apps.” Forbes, Forbes Magazine, 23 Sept. 2020. https://www.forbes.com/?sh=4f17a0972254

Rofagha, Antonia, and Julie De Balilliencourt. "Coming Together to Support Body Positivity on TikTok." Newsroom, TikTok, 16 Aug. 2019. https://newsroom.tiktok.com/en-us/coming-together-to-support-bodypositivity-on-tiktok

Verma, Arushi, and Maria-Irini Avgoulas. "Eating Disorders: Perceptions of Young Women and Social Media Portrayal." International Journal of Health, Wellness \& Society, vol. 5, no. 4, Dec. 2015, pp. 97-105. EBSCOhost. https://doi.org/10.18848/2156-8960/CGP/v05i04/41146

Wides, Sonia Chajet. “TikTok’s Body Image Problem.” The Bardvark, 28 May 2020, https://www.thebardvark.com/

Willen, Claudia. "Lizzo Calls out TikTok for Removing Her Videos in Bathing Suits While Letting Other Women Share Posts in Bikinis: 'I Wonder Why'.." Insider, 6 Mar. 2020. https://www.insider.com/lizzo-tiktokremoving-videos-bikinis-bathing-suits-2020-3 